\title{
Stability Analysis of a Feedback-Controlled Resonant DC-DC Converter
}

\author{
Octavian Dranga, Balázs Buti, and István Nagy, Fellow, IEEE
}

\begin{abstract}
This paper reports on the stability analysis of one member of a dual-channel resonant dc-dc converter family. The study is confined to the buck configuration in symmetrical operation. The output voltage of the converter is controlled by a closed loop applying constant-frequency pulsewidth modulation. The dynamic analysis reveals that a bifurcation cascade develops as a result of increasing the loop gain. The trajectory of the variablestructure piecewise-linear nonlinear system pierces through the Poincaré plane at the fixed point in state space when the loop gain is small. For stability criterion the positions of the characteristic multipliers of the Jacobian matrix belonging to the Poincaré Map Function defined around the fixed point located in the Poincaré Plane is applied. In addition to the stability analysis, a bifurcation diagram is developed showing the four possible states of the feedback loop: the periodic, the quasi-periodic, the subharmonic, and the chaotic states. Simulation and test results verify the theory.
\end{abstract}

Index Terms-Resonant dc-dc converter, stability.

\section{INTRODUCTION}

A FAMILY OF dual-channel resonant dc-dc converters, with 12 members, was introduced in [1] and discussed in a number of other publications later [2]-[4]. The common basic configuration contains two channels, the so-called positive and negative ones. They transfer the power from input to output and are coupled by a resonating capacitor $C$ [2], [3]. The converters can operate in symmetrical mode and in asymmetrical mode when the respective variables in the two channels vary symmetrically (without any energy exchange between channels) and asymmetrically (with energy exchange between channels through the resonating capacitor), respectively. The converters are recommended to be applied in the middle and higher power ranges.

The present investigation is restricted to the buck configuration (Fig. 1) in strictly symmetrical operation and strictly symmetrical load. Its target is not the discussion of the dc-dc resonant converter family but the analysis of the stability of the output voltage control loop built around the buck converter. Two

Manuscript received March 15, 2001; revised May 12, 2002. Abstract published on the Internet September 13, 2002. This work was supported by the Hungarian Research Fund (OTKA TO29026) and the Control Research Group of the Hungarian Academy of Science.

O. Dranga is with The Hong Kong Polytechnic University, Hong Kong, on leave from Budapest University of Technology and Economics, H-1111 Budapest, Hungary.

B. Buti is with the Department of Automation and Applied Informatics, Budapest University of Technology and Economics, H-1111 Budapest, Hungary.

I. Nagy is with the Department of Automation and Applied Informatics, Budapest University of Technology and Economics, H-1111 Budapest, Hungary, and also with the Computer and Automation Institute of the Hungarian Academy of Sciences, H-1111 Budapest, Hungary (e-mail: nagy@elektro.get.bme.hu).

Digital Object Identifier 10.1109/TIE.2002.804983

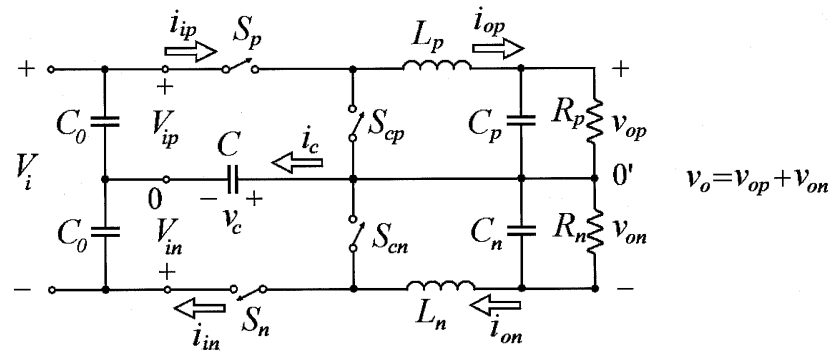

Fig. 1. Resonant buck converter $R_{p}=R_{n} ; C_{p}=C_{n}$.

issues have to be discussed. The first one is the reason beyond the strict symmetry for applying only one voltage control loop to regulate the sum of the output voltages. This issue will be treated in more detail in Section III. The second issue is the explanation of the selection of the exact iterative-map approach using the state-space description of the system. To investigate the stability two main techniques were developed: the timeaverage method and the discrete iterative-map approach. The time-average model essentially ignores the switching details, focusing only on the envelope of the dynamics. The drawback of the time-average method is that as the detailed dynamics within a switching cycle are ignored and only the low-frequency properties are retained, the model gives information only about the low-frequency behavior and its validity will be limited to this range. In order to prove the development of quasi-periodic and chaotic states the time functions of the state variables must be determined, that is, the state-space method must be used. Quasi-periodic and chaotic states are connected to the fast processes and they can be discovered only by following precisely the time histories of the state variables within the switching period without replacing them by their average value. More complete information can be achieved by deriving an iterative function based on the state space trajectory of the system. It expresses the state variables at one sampling instant in terms of the ones belonging to the previous sampling instant. This kind of discrete-time model will be the instrument to detect the bifurcations in the high-frequency range.

Computer simulation studies published in [2] have revealed interesting bifurcation phenomena developing in the course of changing the voltage loop gain. The basics of the method applied in the paper can be found in [6] and [7]. Chaotic behavior of other types of dc-dc converters was investigated in [8]-[15] and chaotic state was treated in drives in [16]-[19]. The general related problem can be read in [20] and [21].

The structure of the paper is as follows. Section II describes the configuration and its basic operation in steady state. Section III summarizes the setup and operation of the output voltage 
control loop. Section IV offers a brief overview of the theoretical background of stability analysis based on the Poincaré Map Function. Section V points out that one switching period consists of two subperiods. It presents the state equations for the two consecutive structures exhibiting one subperiod. It extends the validity of the state equations for the next subperiod due to the periodicity property of the respective configurations. Section VI develops the Poincaré Map Functions and its Jacobian matrix belonging to the fixed point of the periodic solution. Section VII presents the bifurcation diagram. It plots the output voltage sampled at each switching period as a function of a system parameter, gain $K_{V}$, and presents the calculation results of the characteristic multiplier of Jacobian matrix as two of them leaves the unit circle due to the increase of $K_{V}$. Calculation results of quasi-periodic and chaotic states are presented as well. Experimental results in Section VIII verify the theoretical considerations and calculation figures.

\section{CONVERTER CONFIGURATION AND OPERATION}

The buck configuration is shown in Fig. 1. The converter has two channels from input to output, both with two controlled switches and one inductance $L$. Suffixes $i$ and $o$ refer to input and output while suffixes $p$ and $n$ refer to positive and negative channels, respectively. The capacitances across the input terminals shorting high-frequency currents are assumed to be high and they will be neglected in the stability analysis. The configuration version studied in this paper applies only controlled switches (insulated gate bipolar transistors (IGBTs), MOSFETs, or other switches) conducting current in the direction of arrow.

The assumptions used in the description of operation in steady state are as follows: idealized and lossless circuit components, constant and smooth input voltage $V_{i}$ and output voltages, identical load resistance $R$ at the positive and negative sides $\left(R_{p}=R_{n}=R\right)$, neglected commutation times, and symmetrical operation of the two channels. The input voltages $\left(V_{i p}, V_{i n}\right)$, the output voltages $\left(v_{o p}, v_{o n}\right)$, the peak condenser voltages $\left(v_{c p},\left|v_{c n}\right|\right)$, and the commutation angles $\left(\alpha_{p}, \alpha_{n}\right)$ are identical. The asymmetrical operation and asymmetrical load is out of the scope of the current paper.

The controlled switches within one channel are always in complementary states (that is, when $S_{p}$ is on, $S_{c p}$ is off and vice-versa). By turning on switch $S_{p}$, a sinusoidal current pulse $i_{i p}$ is developed from $\omega t=0$ to $\alpha_{p}\left(\omega=2 \pi f_{r}=1 / \sqrt{L C}\right)$ in circuit $S_{p}, L, v_{o p}, C$ and $V_{i p}$ (Fig. 2). (The ripple of $v_{o p}$ and $v_{o n}$ is drawn in Fig. 2(b) for the stability study. Here, ripple-free output voltages are assumed.) The currents are $i_{i p}=i_{o p}=i_{c}$ in interval $0 \leq \omega t \leq \alpha_{p}$. The capacitor voltage $v_{c}$ swings from $v_{c n}$ to $v_{c p}\left(v_{c n}<0\right)$. By turning on switch $S_{c p}$ at $\alpha_{p}$ the choke current commutes from $S_{p}$ to $S_{c p}$ and it circulates in the freewheeling path $S_{c p}, L_{p}$ and $v_{o p}$. The energy stored in the choke at $\omega t=\alpha_{p}$ is depleted in the interval $\alpha_{p}<\omega t<\omega T_{s}$ where $T_{s}=1 / f_{s}$ is the switching period. At discontinuous current conduction (DCM) the stored energy is entirely depleted in interval $\alpha<\omega t<\alpha_{e p}$, where $\alpha_{e p}$ denotes the extinction angle of the inductor current. In DCM the current is zero between $\alpha_{e p}$ and $\omega T_{s}$. In the continuous conduction mode (CCM) of opera-
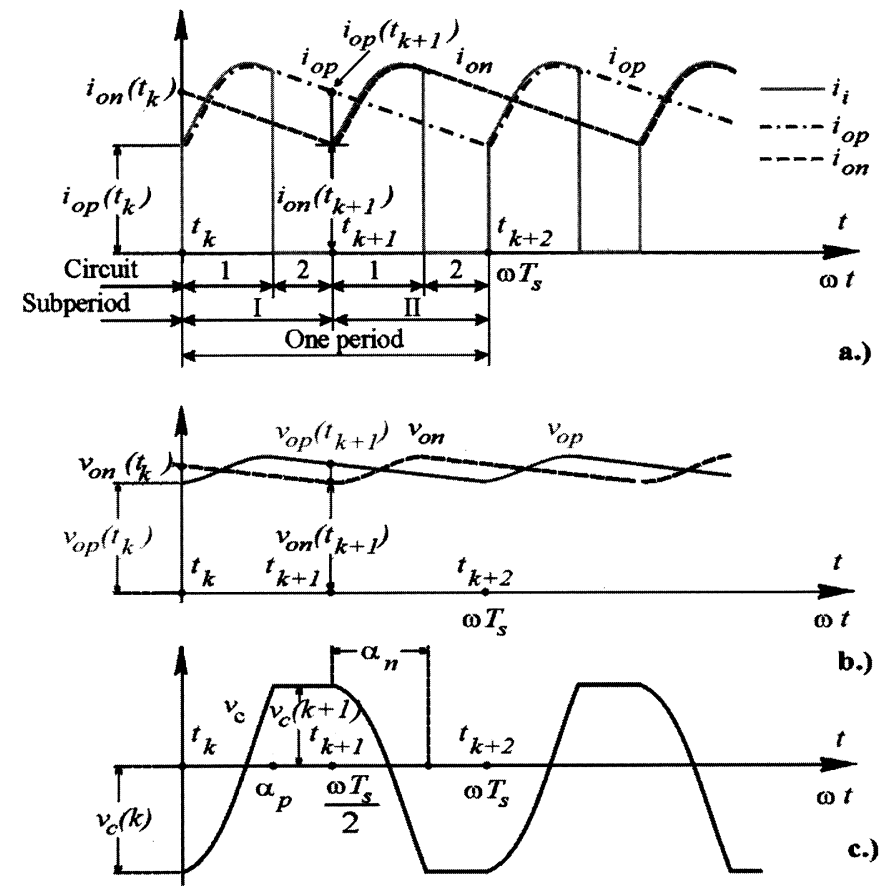

Fig. 2. (a) Time functions of input and output (inductor) currents, (b) output voltages, and (c) condenser voltage in CCM.

tion the inductor current flows continuously $\left(i_{o p}>0\right)$ (Fig. 2). The inductor current $i_{o p}$ decreases both in DCM and in CCM in a linear fashion in interval $\alpha_{p}<\omega t<\omega T_{s}$. After turning on $S_{c p}$ the capacitor voltage $v_{c}$ stops changing. It keeps its value $v_{c p}$ until $\omega T_{s} / 2$.

The same process takes place in the negative channel resulting in a current pulse $i_{o n}$ and negative condenser voltage swing after turning on $S_{n}$ at the beginning of the next half cycle at $\omega T_{s} / 2$ (Fig. 2 ).

The input variables directly set by us are $V_{i}, \alpha, f_{s}$, and $R$. The input voltage $V_{i}$ and the load resistance $R$ are usually given. The selection of the switching angle $\alpha$ and the switching frequency $f_{s}$ depend on the control. Condition $f_{s} \leq f_{r}$ must hold. The reason is that the change of condenser voltage $v_{c}$ in one direction must be completed before its change gets started in the opposite direction. Turning on switch $S_{p}$ and $S_{n}$ must be phase shifted by $\omega T_{s} / 2$, the two input currents may flow only in separate intervals. Furthermore, $\alpha<\omega T_{s} / 2$ must hold.

The output variable determined by the set of input variables is $v_{o}$. The basic steady-state relations are presented in Appendix A.

\section{OutPut Voltage CONTROL}

In order to control the output voltage $v_{o}$ by pulsewidth modulation (PWM) switching a feedback control loop is applied [Fig. 3(a)]. Due to the strictly symmetrical load assumed $\left(R_{p}=\right.$ $R_{n}$ ), one single output voltage control loop provides the control of both $v_{o p}$ and $v_{o n}$ beside $v_{o}$. By applying only one single output load resistance $R$ between the + and - rails and assuming smooth ripple-free $v_{o p}$ and $v_{o n}$ voltages in steady state due to the large capacitances $C_{p}=C_{n}$ as was supposed in Section II, the operation of the converter would be the same as in 


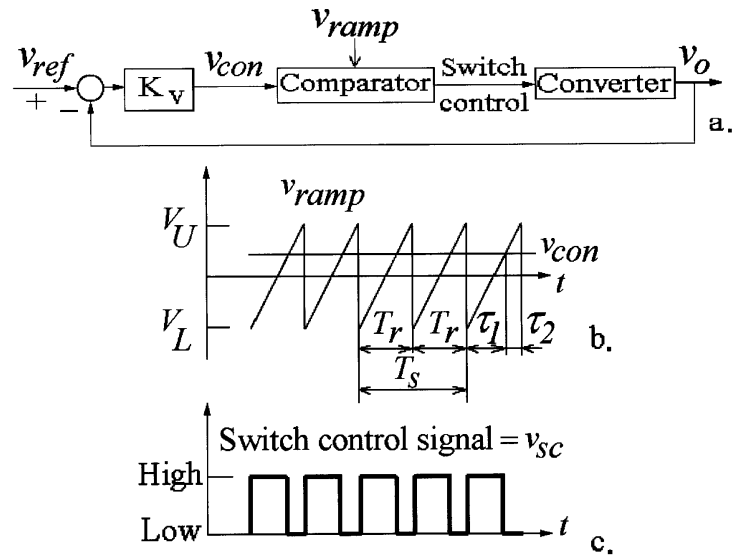

Fig. 3. Feedback loop to control output voltage: (a) block diagram, (b) PWM by ramp wave, and (c) switch control signal.

case of two separate but identical load resistances $\left(R_{p}=R_{n}=\right.$ $R / 2$ ) connected to $v_{o p}$ and $v_{o n}$. Therefore, it is reasonable to presume that the stability analysis carried out for two separate but identical loads will provide similar results as another stability study would offer for a single load resistance, which is out of the scope of the current paper.

The control voltage $v_{\text {con }}$ is obtained by amplifying the error signal $\left(V_{\text {ref }}-v_{o}\right)$ by gain $K_{v} \cdot v_{\text {con }}$ is compared to the repetitive sawtooth waveform [Fig. 3(b)]. Whenever the amplified error signal $v_{\text {con }}$ is greater than the sawtooth waveform, the switch control signal $v_{s c}$ is high [Fig. 3(c)] and the selected controlled switch $S_{p}$ or $S_{n}$ turns on. Otherwise, the switch is off. $S_{p}$ and $S_{n}$ are turned on and off successively, i.e., the switch control signal $v_{s c}$ for $S_{p}$ is generated in one $T_{r}$ period of the sawtooth wave and in the next $T_{r}$ period $v_{s c}$ controls switch $S_{n}$. Hence, the period of the sawtooth wave $T_{r}$ is half of the switching period: $T_{r}=T_{s} / 2$. Note that no switching occurs when $\left|v_{\text {con }}\right|>V_{U}=$ $-V_{L}$ within $T_{r}$.

\section{Theoretical BACKGROUND: Stability OF A PERIODIC SOLUTION}

As presented above, in normal operation the converter exhibits periodic behavior. Periodic time functions describe the waveforms of the state variables with period $T_{s}$ (Figs. 2 and 3).

One of the most useful methods for investigating continuous nonlinear systems involves a discretization technique and by the introduction of the Poincaré map function (PMF) or in short the Poincaré map. For a nonautonomous system the PMF gives the relation between two consecutive points obtained by the sampling of the trajectory evolving in the state space at a rate being equal to the switching frequency $f_{s}$. Assuming that the period of the periodic state is the same as that of the switching frequency, the periodic state corresponds to a fixed point $x^{*}$ denoted by $P$ in the state space. The stability of the periodic state is the same as the stability of the fixed point in the Poincaré map. In the succeeding discussion of stability, "fixed point" can be replaced everywhere by "normal (fundamental) periodic solution."

The Poincaré map function relates the coordinates of consecutive sampled points in state space separated by one switching period $T_{s}$ from each other in time

$$
\underline{x}_{n+1}=\underline{P}\left(\underline{x}_{n}\right)
$$

$(n=1,2 \ldots)$ and in periodic state at the fixed point $\underline{x}^{*}=$ $\underline{P}\left(\underline{x}^{*}\right)$.

Therefore, the stability is determined by the local behavior of PMF near the fixed point $x^{*}$. If all sufficiently small deviations around $\underline{x}^{*}$ tends toward 0 with time, then $\underline{x}^{*}$ and the system are asymptotically stable, the trajectories are attracted to the steady-state trajectory, the limit cycle. $\underline{x}^{*}$ and the limit cycle are unstable if any sufficiently small deviation increases with time and the trajectories moves away from the initial limit cycle [6].

In particular, the local behavior of the PMF in the neighborhood of the fixed point $\underline{x}^{*}$ is governed by its linearization near that fixed point [6]

$$
\Delta \underline{x}_{n+1}=\underline{J}_{n}\left(\underline{x}^{*}\right) \Delta \underline{x}_{n}
$$

where

$$
\underline{J}_{n}\left(\underline{x}^{*}\right)=\left.\frac{d \underline{P}(\underline{x})}{d \underline{x}}\right|_{\underline{x}^{*}}
$$

is the Jacobian matrix of the Poincaré map function $\underline{P}\left(\underline{x}_{n}\right)$, evaluated at the fixed point $\underline{x}^{*}$. Substituting $\underline{J}_{n}\left(\underline{x}^{*}\right)$ by its eigenvalues or characteristic multipliers $\lambda_{i}$ and its right $\underline{u}_{i r}$ and left $\underline{u}_{i l}$ eigenvectors, the linearized relation or the linear map becomes

$$
\Delta \underline{x}_{n+1}=\left(\sum \lambda_{i} \underline{u}_{i r} \underline{u}_{i l}^{T}\right) \Delta \underline{x}_{n} .
$$

The eigenvalues give the amount of expansion and contraction near the periodic solution during one period. Starting from $n=$ 1 , the value $\Delta \underline{x}_{n+1}=\underline{J}_{n}^{n}\left(\underline{x}^{*}\right) \Delta \underline{x}_{1}$, that is,

$$
\Delta \underline{x}_{n+1}=\left(\sum \lambda_{i}^{n} \underline{u}_{i r} \underline{u}_{i l}^{T}\right) \Delta \underline{x}_{1} .
$$

The position of the eigenvalues in the complex plane determines the stability of the fixed point. The fixed point and the limit cycle are asymptotically stable if and only if all eigenvalues in (5) have modulus less than unity, i.e., they lie inside the unit circle; if any eigenvalue has modulus greater than unity, i.e., it lies outside the unit circle, the limit cycle is unstable as $\left|\Delta \underline{x}_{n+1}\right|$ keeps increasing with $n$ due to $\lambda_{i}^{n}$.

\section{State Equations of the Dual-Channel RESONANT CONVERTER}

The studied resonant converter is a variable-structure piecewise-linear nonlinear dynamical system. The structure of the active circuit varies during the periodic steady-state operation (Fig. 2). The structures change periodically during the operation. One period corresponds to the switching cycle $T_{s}$ and it is divided into two subperiods (semiperiods in time) by the repetition of consecutive two structures presented in Fig. 4. The correspondence between the circuits in Fig. 4 and the active parts of the converter configuration (Fig. 1) during one switching period is shown in Table I. The time sequence of structure changes is outlined in Fig. 5. The durations of the same structure in the 


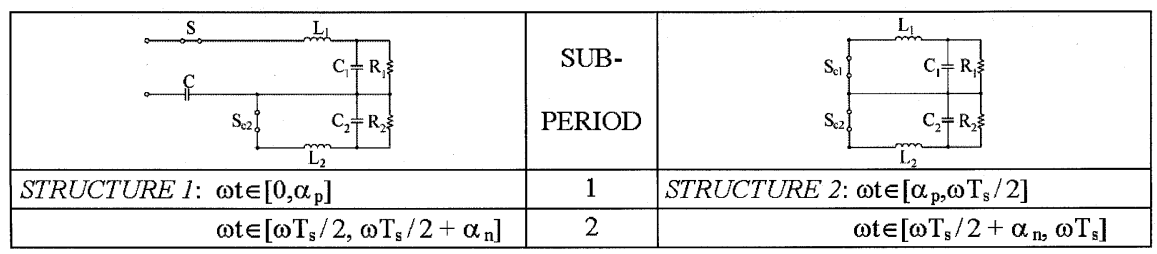

Fig. 4. Converter structures during either subperiod I or subperiod II.

TABLE I Correspondance Between the Converter and the Structures IN FIg. 4 DURING ONE SWITCHING PERIOD

\begin{tabular}{c|c|c|c|c}
\hline \multirow{2}{*}{} & \multicolumn{3}{|c}{ ONE SWITCHING PERIOD } \\
\cline { 2 - 5 } & \multicolumn{2}{|c|}{ SUBPERIOD I } & \multicolumn{2}{c}{ SUBPERIOD II } \\
\cline { 2 - 5 } & STRUCTURE 1 & STRUCTURE 2 & STRUCTURE 1 & STRUCTURE 2 \\
\hline$S$ & $S_{0}$ & - & $S_{n}$ & - \\
\hline$S_{c 1}$ & - & $S_{c p}$ & - & $S_{c n}$ \\
\hline$S_{c 2}$ & $S_{c n}$ & $S_{c n}$ & $S_{c p}$ & $S_{c p}$ \\
\hline$L_{1}$ & $L_{p}$ & $L_{p}$ & $L_{n}$ & $L_{n}$ \\
\hline$L_{2}$ & $L_{n}$ & $L_{n}$ & $L_{p}$ & $L_{p}$ \\
\hline$C_{1}$ & $C_{p}$ & $C_{p}$ & $C_{n}$ & $C_{n}$ \\
\hline$C_{2}$ & $C_{n}$ & $C_{n}$ & $C_{p}$ & $C_{p}$ \\
\hline$R_{1}$ & $R_{p}$ & $R_{p}$ & $R_{n}$ & $R_{n}$ \\
\hline$R_{2}$ & $R_{n}$ & $R_{n}$ & $R_{p}$ & $R_{p}$ \\
\hline
\end{tabular}

two subperiods are equal in periodic state due to the symmetrical operation investigated: $\alpha_{p}=\alpha_{n}=\alpha$.

Since each structure has linear dynamics, the resonant converter is a piecewise-linear system. On the other hand, the whole system, the feedback controlled converter is nonlinear due to the dependence of commutation angles $\alpha_{p}, \alpha_{n}$ on the state variable $v_{o}=v_{o p}+v_{o n}$. The linear state equations for structure 1 in subperiod I using Table I are given in (6) and in dimensionless form in (7)

$$
\begin{aligned}
\frac{d v_{o p}}{d t} & =-\frac{1}{R_{p} C_{p}} v_{o p}+\frac{1}{C_{p}} i_{o p} \\
\frac{d v_{o n}}{d t} & =-\frac{1}{R_{n} C_{n}} v_{o n}+\frac{1}{C_{n}} i_{o n} \\
\frac{d v_{c}}{d t} & =\frac{1}{C} i_{o p} \\
\frac{d i_{o p}}{d t} & =-\frac{1}{L_{p}} v_{o p}-\frac{1}{L_{p}} v_{c}+\frac{1}{L_{p}} v_{i p} \\
\frac{d i_{o n}}{d t} & =-\frac{1}{L_{n}} v_{o n} \\
\frac{d x_{1}}{d \tau} & =-a x_{1}+b x_{4} \\
\frac{d x_{2}}{d \tau} & =-a x_{2}+b x_{5} \\
\frac{d x_{3}}{d \tau} & =\pi x_{4} \\
\frac{d x_{4}}{d \tau} & =-\pi\left(x_{1}+x_{3}-1\right) \\
\frac{d x_{5}}{d \tau} & =-\pi x_{2}
\end{aligned}
$$

where

$$
\begin{aligned}
x_{1} & =\frac{v_{o p}}{\frac{V_{i}}{2}} \\
x_{2} & =\frac{v_{o n}}{\frac{V_{i}}{2}} \\
x_{3} & =\frac{v_{c}}{\frac{V_{i}}{2}} \\
x_{4} & =\frac{Z \cdot i_{o p}}{\frac{V_{i}}{2}} \\
x_{5} & =\frac{Z \cdot i_{o n}}{\frac{V_{i}}{2}} \\
\tau & =\frac{t}{T_{r}} \\
a & =\frac{T_{r}}{T_{o}} \\
b & =\frac{\pi T_{c}}{T_{o}}=\frac{\pi C}{C_{o}} \\
T_{o} & =R C_{o} \\
T_{C} & =R C, \\
Z & =\sqrt{\frac{L}{C}}
\end{aligned}
$$

and $V_{i} / 2=V_{i p}=V_{i n}, L=L_{p}=L_{n}, C_{o}=C_{p}=C_{n}$, $R=R_{p}=R_{n}$, and $T_{r}=T_{s} / 2=\pi \sqrt{L C}$. The state equation of structure 1 in matrix form is

$$
\begin{gathered}
\underline{\dot{x}}=\underline{A}_{1} \cdot \underline{x}+\underline{B}_{1}, \quad \underline{x}=\left[\begin{array}{l}
x_{1} \\
x_{2} \\
x_{3} \\
x_{4} \\
x_{5}
\end{array}\right] ; \\
\underline{A}_{1}=\left[\begin{array}{ccccc}
-a & 0 & 0 & b & 0 \\
0 & -a & 0 & 0 & b \\
0 & 0 & 0 & \pi & 0 \\
-\pi & 0 & -\pi & 0 & 0 \\
0 & -\pi & 0 & 0 & 0
\end{array}\right] ; \underline{B}_{1}=\left[\begin{array}{l}
0 \\
0 \\
0 \\
\pi \\
0
\end{array}\right] .
\end{gathered}
$$

State equations for structure 2 in subperiod I are given in (10) and they are presented in matrix form in (11)

$$
\begin{aligned}
\frac{d v_{o p}}{d t} & =-\frac{1}{R_{p} C_{p}} v_{o p}+\frac{1}{C_{p}} i_{o p} \\
\frac{d v_{o n}}{d t} & =-\frac{1}{R_{n} C_{n}} v_{o n}+\frac{1}{C_{n}} i_{o n} \\
\frac{d v_{c}}{d t} & =0 \\
\frac{d i_{o p}}{d t} & =-\frac{1}{L_{p}} v_{o p} \\
\frac{d i_{o n}}{d t} & =-\frac{1}{L_{n}} v_{o n}
\end{aligned}
$$




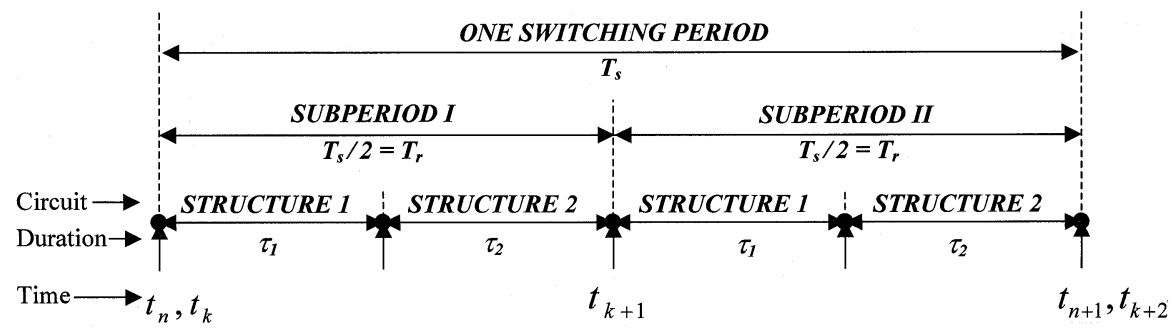

Fig. 5. Time sequence of structure changes.

$$
\begin{aligned}
\underline{\dot{x}} & =\underline{A}_{2} \cdot \underline{x} \\
\underline{x} & =\left[\begin{array}{lllll}
x_{1} & x_{2} & x_{3} & x_{4} & x_{5}
\end{array}\right]^{T} \\
\underline{A}_{2}= & {\left[\begin{array}{ccccc}
-a & 0 & 0 & b & 0 \\
0 & -a & 0 & 0 & b \\
0 & 0 & 0 & 0 & 0 \\
-\pi & 0 & 0 & 0 & 0 \\
0 & -\pi & 0 & 0 & 0
\end{array}\right] }
\end{aligned}
$$

Due to the periodicity the following relations hold in steady state among the end and start values of the state variables in each subperiod (Fig. 2): $v_{o p, e}=v_{o n, s} ; v_{o n, e}=v_{o p, s} ; v_{c, e}=-v_{c, s}$; $i_{o p, e}=i_{o n, s} ; i_{o n, e}=i_{o p, s}$ where suffixes $e$ and $s$ stands for end and start, respectively. In short,

$$
\underline{x}_{e}=\underline{T x}_{s}
$$

where the periodicity or transfer matrix

$$
\underline{T}=\underline{T}^{-1}=\left[\begin{array}{ccccc}
0 & 1 & 0 & 0 & 0 \\
1 & 0 & 0 & 0 & 0 \\
0 & 0 & -1 & 0 & 0 \\
0 & 0 & 0 & 0 & 1 \\
0 & 0 & 0 & 1 & 0
\end{array}\right]
$$

Multiplication of the starting value of the state variable $\underline{x}_{S}$ by $\underline{T}$ transforms $\underline{x}_{s}$ in time forward to the end of the subperiod to calculate $\underline{x}_{e}$. On the other hand, the equation

$$
\underline{x}_{s}=\underline{T}^{-1} \underline{x}_{e}
$$

obtained from (12) can be interpreted as a backward transformation in time to calculate $\underline{x}_{s}$ from $\underline{x}_{e}$.

By utilizing the periodicity of the structure series explained in Figs. 4 and 5 and in Table I, even in transient $\underline{x}_{k, e}$ can be transformed backward to calculate $\underline{x}_{k+1, s}$

$$
\underline{x}_{k+1, s}=\underline{T}^{-1} \underline{x}_{k, e}
$$

where $k$ denotes the $k$ 'th subperiod. $\underline{x}_{k+1, s}$ can be used as initial value of the subperiod $k+1$ for structure 1 and the same state (9) and (11) can be applied in subperiod $k+1$ as the ones used in subperiod $k$ due to the periodicity of the structures.

As was mentioned above, the system within each subperiod is piecewise linear. The PWM-controlled feedback converter becomes a nonlinear system, due to the dependence of the structure change instant $\tau_{1}$ (the turn-on time of the switch $S_{c p}$ and $S_{c n}$ ) on the state variable, the output voltage.

\section{Stability AnALysis}

\section{A. Introduction}

It has been stated in Section IV that the stability of the feedback control loop including the converter in periodic operation is determined by the eigenvalues of the Jacobian matrix $\underline{J}_{n}\left(\underline{x}^{*}\right)$ of the Poincaré map function at the corresponding fixed point $\underline{x}^{*}[(3)]$. It has been shown in the previous section that the dynamics of one switching period can be described by applying the state equation (9) and (11) on two consecutive steps for subperiod I but before applying them in the second time the end values of state variables $\underline{x}_{k, e}$ must be transformed backward by using transformation matrix $\underline{T}^{-1}[(15)]$.

The first target is the determination of the Poincaré map function (PMF) $\underline{P}\left(\underline{x}^{*}\right)$. The starting value of the five state variables $\underline{x}_{s}$ at the start of the switching period $T_{S}$ in steady state is chosen at fixed point $\underline{x}^{*}=\underline{x}_{s}$. Changing $\underline{x}_{s}$ by small deviation $\Delta \underline{x}_{1, s}=\Delta \underline{x}_{0}$, the end value of the state vector at the $k$ th subperiod is

$$
\underline{x}_{k, e}=\underline{f}\left(\underline{x}_{k, s}\right)
$$

where $k=1,2, \ldots$ is the number of subperiods. Function $\underline{f}$ can easily be determined first by solving state (9) and after state (11) provided that, $\underline{x}_{k, s}$ and $\tau_{\kappa, 1}$ are known. Interval $\tau_{\kappa, 1}$ lasts as long as switch $S_{p}$ (or $S_{n}$ ) is on (Fig. 5). $\tau_{\kappa, 1}$ is a function of $\underline{x}_{k, s}$, that is, $\tau_{\kappa, 1}\left(\underline{x}_{k, s}\right)$. After transforming $\underline{x}_{k, e}$ backward, the starting value of the state vector in the next subperiod $k+1$ is obtained [(15)]

$$
\underline{x}_{k+1, s}=\underline{T}^{-1} \underline{x}_{k, e}=\underline{T}^{-1} \underline{f}\left(\underline{x}_{k, s}\right) .
$$

The equations and the procedure is the same in subperiod $k+1$ as in subperiod $k$

$$
\underline{x}_{k+2, s}=\underline{T}^{-1} \underline{f}\left(\underline{x}_{k+1, s}\right)=\underline{T}^{-1} \underline{f}\left(\underline{T}^{-1} \underline{f}\left(\underline{x}_{k, s}\right)\right) .
$$

Equation (18) is the PMF of one switching period (Fig. 5) $\left(\underline{x}_{n, s}=\underline{x}_{k, s}\right)$

$$
\underline{x}_{n+1, s}=\underline{P}_{n}\left(\underline{x}_{n, s}\right) .
$$

On the other hand, (17) is the PMF of one subperiod

$$
\underline{x}_{k+1, s}=\underline{P}_{k}\left(\underline{x}_{k, s}\right) .
$$

In steady state $\underline{x}^{*}=\underline{P}_{n}\left(\underline{x}^{*}\right)=\underline{P}_{\underline{k}}\left(\underline{x}^{*}\right)$. As the PMF is the same in each subperiod the dynamics of the system is fully described by $\underline{P}_{k}\left(\underline{x}^{*}\right)$. Conclusion for stability can be drawn either from $\underline{P}_{n}$ or $\underline{P}_{k}$. 
For small deviation around the fixed point by linearizing $\underline{P}_{k}\left(\underline{x}^{*}\right)$ in the neighborhood of $\underline{x}^{*}$ in subperiod 1 [(3), (4)]

$$
\Delta \underline{x}_{k+1, s}=\underline{J}_{k}\left(\underline{x}^{*}\right) \Delta \underline{x}_{k, s}=\underline{J}_{k}\left(\underline{x}^{*}\right) \Delta \underline{x}_{n, s}
$$

and in the next subperiod

$$
\Delta \underline{x}_{n+1, s}=\Delta \underline{x}_{k+2, s}=\underline{J}_{k}\left(\underline{x}^{*}\right) \Delta \underline{x}_{k+1, s}=\underline{J}_{k}^{2}\left(\underline{x}^{*}\right) \Delta \underline{x}_{n, s}
$$

where $\underline{J}_{k}\left(\underline{x}^{*}\right)$ is the Jacobian matrix of $\underline{P}_{k}\left(\underline{x}^{*}\right)$

$$
\underline{J}_{k}\left(\underline{x}^{*}\right)=\left.\frac{d \underline{P} k(\underline{x})}{d \underline{x}}\right|_{\underline{x}^{*}} .
$$

From (22), the relation between the two Jacobian matrices

$$
\underline{J}_{n}\left(\underline{x}^{*}\right)=\underline{J}_{k}^{2}\left(\underline{x}^{*}\right) \text {. }
$$

The absolute values of the eigenvalues of $\underline{J}_{k}$ or $\underline{J}_{n}$ must be smaller than 1 for stable operation [(5)]. The eigenvalues of $\underline{J}_{k}$ are used for the stability study and a Matlab program was developed to compute them.

\section{B. Determination of $\underline{P}_{k}\left(\underline{x}^{*}\right)$}

First, function $\underline{f}\left(\underline{x}_{k, s}\right)$ is determined [see (16)]. The solution of state equation (9) is

$$
\underline{x}_{k}\left(\delta_{k, 1}\right)=\underline{W}_{1}\left(\delta_{k, 1}\right) \underline{x}_{k, s}+\left[\underline{W}_{1}\left(\delta_{k, 1}\right)-I\right] \underline{A}_{1}^{-1} \underline{B}_{1}
$$

and that of state (11) is

$$
\underline{x}_{k, e}=\underline{W}_{2}\left(1-\delta_{k, 1}\right) \underline{x}_{k}\left(\delta_{k, 1}\right)
$$

where $\delta_{k, 1}=\tau_{k, 1} / T_{r} ; \delta_{k, 2}=\tau_{k, 2} / T_{r}=1-\delta_{k, 1}$

$$
\begin{aligned}
\underline{W}_{1}\left(\delta_{k, 1}\right) & =e^{\underline{A}_{1} \delta_{k, 1}} ; \\
\underline{W}_{2}\left(1-\delta_{k, 1}\right) & =e^{\underline{A}_{2}\left(1-\delta_{k, 1}\right)}
\end{aligned}
$$

are the transition matrixes of the two structures, respectively, as well as one particular solution $\underline{x}_{p}=-\underline{A}_{1}^{-1} \cdot \underline{B}$ is obtained from (9) by substituting $\underline{\dot{x}}=0$. By using (9) and calculating $\underline{x}_{p}=-\underline{A}_{1}^{-1} \cdot \underline{B}_{1}=-\operatorname{adj} \underline{A}_{1} /\left|\underline{A}_{1}\right| \cdot \underline{B}_{1}$, the result is: $x_{1}=0$, $x_{2}=0, x_{3}=\left(v_{c}\right) /\left(v_{i} / 2\right)=1, x_{4}=0$ and $x_{5}=0$. This result as one possible particular solution can easily be deduced by physical consideration without any calculation as well. $\underline{I}$ is the identity matrix. The determination of $\delta_{k, 1}$ will be treated later.

By applying in cascade the solutions of differential equation (9) and (11), Poincaré map function $\underline{P}_{k}$ and function $f$ from (16), (17), (20), (25), and (26)

$$
\begin{aligned}
\underline{x}_{k+1, s}= & \underline{P}_{k}\left(\underline{x}_{k, s}\right)=\underline{T}^{-1} \underline{x}_{k, e}=\underline{T}^{-1} \underline{f}\left(\underline{x}_{k, s}\right) \\
= & \underline{T}^{-1} \underline{W}_{2}\left(1-\delta_{k, 1}\right) \\
& \cdot\left\{\underline{W}_{1}\left(\delta_{k, 1}\right) \cdot \underline{x}_{k, s}+\left[\underline{W}_{1}\left(\delta_{k, 1}\right)-\underline{I}\right] \cdot \underline{A}_{1}^{-1} \cdot \underline{B}_{1}\right\} .
\end{aligned}
$$

In periodic steady state, $\underline{x}_{k+1, s}=\underline{x}_{k, s}=\underline{x}^{*}$.

\section{Jacobian Matrix $\underline{J}_{k}\left(\underline{x}^{*}\right)$}

The next step is the determination of the Jacobian matrix $\underline{J}_{k}$ [(23)]. The basic equations needed are (28) and (27) where $\underline{x}_{k, s}=\underline{x}$ and $\delta_{k, 1}=\delta_{1}$ have to be substituted and the derivative of $\underline{P}_{k}$ has to be evaluated at the fixed point $\underline{x}^{*} . \delta_{1}\left(\underline{x}^{*}\right)$ belongs to $\underline{x}^{*}$ and determined by $\underline{x}^{*}$. As $f\left(\underline{x}^{*}\right)$ is expressed in (28) as a function of $\underline{x}^{*}$ and $\delta_{1}\left(\underline{x}^{*}\right)$ the Jacobian matrix

$\underline{T} \cdot \underline{J}_{k}\left(\underline{x}^{*}\right)=\left.\frac{d \underline{f}\left(\underline{x}, \delta_{1}(\underline{x})\right)}{d \underline{x}}\right|_{\underline{x}^{*}}=\left.\left[\frac{\partial \underline{f}}{\partial \underline{x}}+\frac{\partial \underline{f}}{\partial \delta_{1}}\left(\frac{d \delta_{1}}{d \underline{x}}\right)^{T}\right]\right|_{\underline{x}^{*}}$.

Two out of three partial derivatives can be calculated from (28) and (27)

$$
\begin{aligned}
& \left.\frac{\partial \underline{f}}{\partial \underline{x}}\right|_{\underline{x}^{*}}=\underline{W}_{2}\left(1-\delta_{1}\right) \cdot \underline{W}_{1}\left(\delta_{1}\right) \\
& \left.\frac{\partial \underline{f}}{\partial \delta_{1}}\right|_{\underline{x}^{*}}=\frac{\partial \underline{W_{2}\left(1-\delta_{1}\right)}}{\partial \delta_{1}} \\
& \cdot\left\{\underline{W}_{1}\left(\delta_{1}\right) \cdot \underline{x}^{*}+\left[\underline{W}_{1}\left(\delta_{1}\right)-\underline{I}\right] \cdot \underline{A}_{1}^{-1} \cdot \underline{B}_{1}\right\} \\
& +\underline{W}_{2}\left(1-\delta_{1}\right) \\
& \cdot \frac{\partial\left\{\underline{W}_{1}\left(\delta_{1}\right) \cdot \underline{x}^{*}+\left[\underline{W}_{1}\left(\delta_{1}\right)-\underline{I}\right] \cdot \underline{A}_{1}^{-1} \cdot \underline{B}_{1}\right\}}{\partial \delta_{1}} \\
& =-\underline{W}_{2}\left(1-\delta_{1}\right) \cdot \underline{A}_{2} \cdot \underline{x}\left(\delta_{1}\right)+\underline{W}_{2}\left(1-\delta_{1}\right) \cdot \frac{\partial \underline{x}\left(\delta_{1}\right)}{\partial \delta_{1}} \\
& =\underline{W}_{2}\left(1-\delta_{1}\right) \cdot\left(-\underline{\dot{x}}_{2, s}+\underline{\dot{x}}_{1, e}\right)
\end{aligned}
$$

where $\underline{\dot{x}}_{1, e}$ and $\underline{\dot{x}}_{2, s}$ are the velocities of state vector $\underline{x}$ at the end of structure 1 [(9)], and at the beginning of structure 2 [(11)], respectively.

To determine $\left(d \delta_{1} / d \underline{x}\right)^{T}$ the relation between $\delta_{1}$ and $\underline{x}$ is needed.

The PWM control terminates the structure 1 in subperiod 1 at $\delta_{1}$ when the difference between the control voltage $v_{\text {con }}$ and the sawtooth waveform $v_{\text {ramp }}$ becomes zero (Fig. 3). In dimensionless form in steady state,

$$
\begin{aligned}
\left.e\left(\underline{x}, \delta_{1}\right)\right|_{\underline{x}^{*}}= & \frac{v_{\mathrm{con}}\left(\delta_{1}\right)-v_{\mathrm{ramp}}\left(\delta_{1}\right)}{\frac{V_{i}}{2}} \\
= & K_{V} \cdot\left[V_{\mathrm{ref}}^{*}-x_{1}\left(\delta_{1}\right)-x_{2}\left(\delta_{1}\right)\right] \\
& -V_{L}^{*}-\left(V_{U}^{*}-V_{L}^{*}\right) \cdot \delta_{1}= \\
= & K_{V} \cdot V_{\mathrm{ref}}^{*}-\underline{k}^{T} \cdot \underline{x}\left(\delta_{1}\right)-V_{L}^{*}-\left(V_{U}^{*}-V_{L}^{*}\right) \cdot \delta_{1}= \\
= & K_{V} \cdot V_{\mathrm{ref}}^{*}-\underline{k}^{T} \cdot\left\{\underline{W}_{1}\left(\delta_{1}\right) \cdot \underline{x}\right. \\
& \left.+\left[\underline{W}_{1}\left(\delta_{1}\right)-\underline{I}\right] \cdot \underline{A}_{1}^{-1} \cdot \underline{B}_{1}\right\} \\
& -V_{L}^{*}-\left(V_{U}^{*}-V_{L}^{*}\right) \cdot \delta_{1}=0
\end{aligned}
$$

where $V_{\text {ref }}^{*}=V_{\text {ref }} /\left(V_{i} / 2\right), V_{L}^{*}=V_{L} /\left(V_{i} / 2\right), V_{U}^{*}=$ $V_{U} /\left(V_{i} / 2\right)$, and $\underline{k}^{T}=\left[\begin{array}{lllll}K_{V} & K_{V} & 0 & 0 & 0\end{array}\right]$.

Relations (8) and (25) were used in (32).

The derivative $\left(d \delta_{1} / d \underline{x}\right)^{T}$ can be calculated from (32). For small changes $(\partial e / \partial \underline{x})^{T} d \underline{x}+\left(\partial e / \partial \delta_{1}\right) d \delta_{1}=0$ and

$$
\left(\frac{\partial \delta_{1}}{\partial \underline{x}}\right)_{\underline{x}^{*}}^{T}=-\left(\frac{\partial e}{\partial \delta_{1}}\right)^{-1}\left(\frac{\partial e}{\partial \underline{x}}\right)^{T} .
$$


The two partial derivatives from (32) are

$$
\begin{aligned}
\left.\frac{\partial e}{\partial \delta_{1}}\right|_{\underline{x}^{*}}= & -\underline{k}^{T} \\
& \cdot \frac{\partial\left\{\underline{W}_{1}\left(\delta_{1}\right) \cdot \underline{x}+\left[\underline{W}_{1}\left(\delta_{1}\right)-\underline{I}\right] \cdot \underline{A}_{1}^{-1} \cdot \underline{B}_{1}\right\}}{\partial \delta_{1}} \\
& -\left(V_{U}^{*}-V_{L}^{*}\right)=-\underline{k}^{T} \cdot \frac{\partial \underline{x}\left(\delta_{1}\right)}{\partial \delta_{1}}-\left(V_{U}^{*}-V_{L}^{*}\right) \\
= & -\underline{k}^{T} \cdot \underline{\dot{x}}_{1, e}-\left(V_{U}^{*}-V_{L}^{*}\right) \\
\left(\frac{\partial e}{\partial \underline{x}}\right)_{\underline{x}^{*}}^{T}= & -\underline{k}^{T} \cdot \underline{W}_{1}\left(\delta_{1}\right) .
\end{aligned}
$$

Substituting (33) into (29),

$$
\underline{T J}_{k}\left(\underline{x}^{*}\right)=\left.\left[\frac{\partial \underline{f}}{\partial \underline{x}}-\frac{\partial \underline{f}}{\partial \delta_{1}}\left(\frac{\partial e}{\partial \delta_{1}}\right)^{-1}\left(\frac{\partial e}{\partial \underline{x}}\right)^{T}\right]\right|_{\underline{x}^{*}} .
$$

Substituting all derivatives, that is, (30), (31), (34), and (35) into (36), the Jacobian matrix

$$
\begin{aligned}
\underline{J}_{k}\left(\underline{x}^{*}\right)= & \underline{T}^{-1} \cdot\left[\underline{W}_{2}\left(1-\delta_{1}\right) \cdot \underline{W}_{1}\left(\delta_{1}\right)\right. \\
& \left.-\frac{\underline{W}_{2}\left(1-\delta_{1}\right) \cdot\left(\underline{x}_{1, e}-\underline{x}_{2, s}\right) \cdot \underline{k}^{T} \cdot \underline{W}_{1}\left(\delta_{1}\right)}{\underline{k}^{T} \cdot \underline{\dot{x}}_{1, e}+V_{U}^{*}-V_{L}^{*}}\right] \\
= & \underline{T}^{-1} \cdot \underline{W}_{2}\left(1-\delta_{1}\right) \\
& \cdot\left[\underline{I}-\frac{\underline{x}_{1, e}-\underline{\dot{x}}_{2, s}}{\underline{k}^{T} \cdot \underline{\dot{x}}_{1, e}+V_{U}^{*}-V_{L}^{*}} \underline{k}^{T}\right] \\
& \cdot \underline{W}_{1}\left(\delta_{1}\right)=\underline{T}^{-1} \cdot \underline{W}_{2}\left(1-\delta_{1}\right) \cdot \underline{M} \cdot \underline{W}_{1}\left(\delta_{1}\right) .
\end{aligned}
$$

The calculation of matrix

$$
M \underline{\underline{x}}_{\underline{*}^{*}}=\left.\left[\underline{I}-\frac{\underline{\dot{x}}_{1, e}-\underline{\dot{x}}_{2, s}}{\underline{k}^{T} \cdot \underline{\dot{x}}_{1, e}+V_{U}-V_{L}} \underline{k}^{T}\right]\right|_{\underline{x}^{*}}
$$

requires the velocity of state vector at the end of structure 1 $\left(\underline{\dot{x}}_{1, e}\right)$ and that at the start of structure $2\left(\underline{\dot{x}}_{2, s}\right)$, which are defined by the state models of the two structures, by (9) and (11), respectively. The state vector $\underline{x}\left(\delta_{1}\right)$ is needed for the calculation both for $\underline{\dot{x}}_{1, e}=\underline{A}_{1} \cdot \underline{x}\left(\delta_{1}\right)+\underline{B}_{1}$ and $\underline{\dot{x}}_{2, s}=\underline{A}_{2} \cdot \underline{x}\left(\delta_{1}\right)$. It can be determined from the solution of (9) [see (28)]

$$
\underline{x}\left(\delta_{1}\right)=\underline{W}_{1}\left(\delta_{1}\right) \cdot \underline{x}^{*}+\left[\underline{W}_{1}\left(\delta_{1}\right)-\underline{I}\right] \cdot \underline{A}_{1}^{-1} \cdot \underline{B}_{1} .
$$

Knowing all terms in matrix $\underline{J}_{k}$, its eigenvalues can be calculated, e.g., by Matlab.

Matrix $\underline{M}$ takes into account the sudden change in the velocity of the state vector at the switching instant $\delta_{1}$ from $\underline{\dot{x}}_{1, e}$ to $\underline{\dot{x}}_{2, s}$. If $\underline{\dot{x}}_{1, e}=\underline{\dot{x}}_{2, s}$, then $\underline{M}=\underline{I}$.

\section{Determination of $\delta_{1}$}

To calculate the eigenvalues of $\underline{J}_{k}$ the value $\delta_{1}$ must be determined. An iterative algorithm was used to this end. The initial value $\delta_{1, i n}$ was obtained from the steady-state relations derived in Appendix A, where the filter capacitor at the output was assumed to be very large, as it is normally the case in applications requiring a nearly ripple-free instantaneous output voltage $V_{o}$. The corresponding steady-state duty ratio $\delta_{1, \text { in }}$ was determined by the PWM control [Fig. 3(b)]

$$
\delta_{1, i n}=\frac{\alpha}{\pi}=\frac{K_{v}\left(V_{\mathrm{ref}}-V_{o}\right)-V_{L}}{V_{U}-V_{L}} .
$$

The average output voltage $V_{o}$ can be expressed from(a9) by taking into consideration that $f_{s} / f_{r}=1$

$$
V_{o}=\frac{V_{i}}{1+\frac{\pi-\frac{\alpha}{2}}{t g\left(\frac{\alpha}{2}\right)}} .
$$

Substituting $V_{o}$ from (41) into (40), $\delta_{1, \text { in }}=\tau_{1} / T_{r}=\alpha / \pi$ was calculated from the transcendental equation.

The accurate value $\delta_{1}$ deviates from $\delta_{1, i n}$ due to the ripple in $v_{o}$. It has been shown that in periodic steady-state $\underline{x}^{*}=$ $\underline{T}^{-1} \cdot f\left(\underline{x}^{*}, \delta_{1, a c}\right)$, where $\delta_{1, a c}$ is the accurate value giving the accurate meeting point of $v_{\text {con }}$ and $v_{\text {ramp }}$ within period $T_{r}$ [Fig. 3(b)], that is, satisfying (32). The iteration for the calculation of $\delta_{1, a c}$ starts by substituting $\delta_{1, i n}$ in (28) and calculating $\underline{x}_{1, s}$, that is, the first approximation of the time function $v_{o}$ or $v_{\text {con }}$ or in general $\underline{x}_{k, s}$ in the $k$ th iteration step from relation

$$
\begin{aligned}
\underline{x}_{k}=\left[\underline{T}-\underline{W}_{2}\left(1-\delta_{k, 1}\right) \cdot \underline{W}_{1}\left(\delta_{k, 1}\right)\right]^{-1} \\
\quad \underline{W}_{2}\left(1-\delta_{k, 1}\right) \cdot\left[\underline{W}_{1}\left(\delta_{k, 1}\right)-\underline{I}\right] \cdot \underline{A}_{1}^{-1} \cdot \underline{B}_{1} .
\end{aligned}
$$

Equation (42) was derived from (28) by assuming steady state, that is, $\underline{x}_{k, s}=\underline{x}_{k+1, s}$. Knowing $v_{\text {con }}$, the meeting

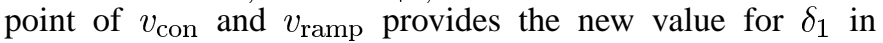
the next iteration step. By setting a limit $\varepsilon$ for the deviation in $\delta_{1}$ in two consecutive iteration steps in such a way that $\left|\delta_{k+1,1}-\delta_{k, 1}\right| / \delta_{k+1,1}<\varepsilon, \delta_{1, a c}$ can be approximated at will, where $k$ is the number of iteration steps.

\section{CAlculation Results}

\section{A. Bifurcation Diagram}

One of the most useful ways for the presentation of the great amount of information obtained by simulation or test and describing the complex behavior of nonlinear dynamic system is the bifurcation diagram. Selecting one single state variable for observation, e.g., the sampled output voltage $v_{O}$, the diagram records its change as a function of one system parameter, e.g., $K_{V}$ over its range of interest in steady state. To generate the bifurcation diagram of the feedback converter configuration studied, the output voltage $v_{o}$ was sampled and stored at the start of every switching cycle $\left(v_{o k}=v_{o}\left(k T_{s}\right)\right)$. The data $v_{o k}$ were collected only after the initial transient is over.

A bifurcation diagram of the feedback converter showing its behavior in a wide variation range of the gain $K_{V}$ is presented in Fig. 6. Starting from left, the bifurcation diagram reveals the stable period-1 domain (Fig. 2), since in this region there is just one single sampled value $\left[v_{o k}=v_{o}\left(k T_{s}\right)\right]$ for a given gain $K_{V}$, i.e., the output voltage $v_{o}$ repeats itself in each switching period $T_{s}$. The first bifurcation, shown in magnified form in Fig. 7, takes place about $K_{V}=3.5$ and, in fact, it is the subject of the stability analysis just deduced. 


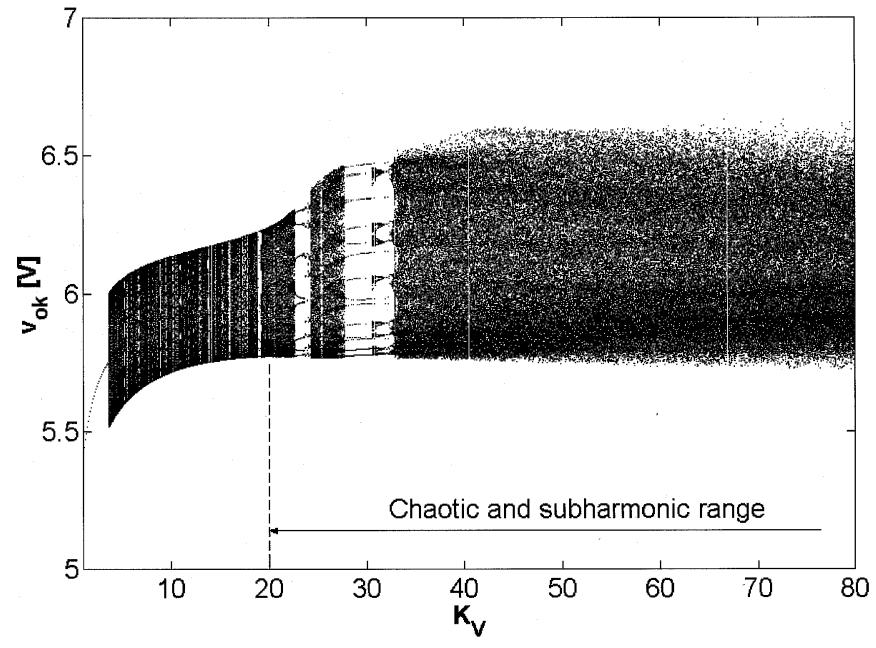

Fig. 6. Bifurcation diagram.

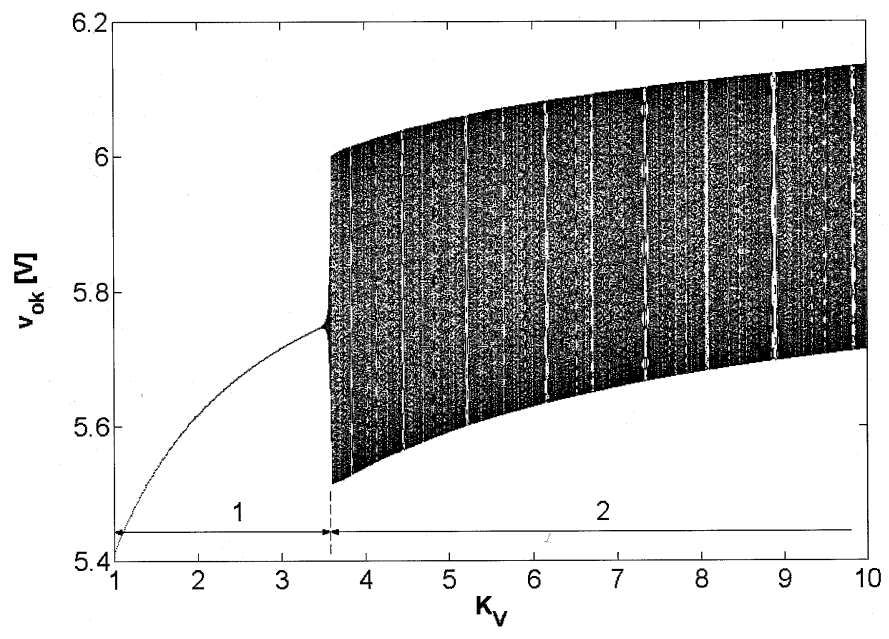

Fig. 7. Enlarged part of the bifurcation diagram (1: periodic range; 2: quasi-periodic range).

\section{B. Loci of the Characteristic Multipliers}

They are presented in Fig. 8 for three values of the voltage gain: $K_{v}=3$ with "+" mark, $K_{v}=3.6$ with "o" mark, and $K_{v}=4$ with "x" mark. It can clearly be seen that a complex-conjugate pair of characteristic multipliers passes through the unit circle as the feedback loop gain is increased. The numerical values are listed in Table II and it denotes a Hopf bifurcation. According to the stability condition, as long as the characteristic multipliers lie inside the unit circle, the periodic steady state solution is asymptotically stable. When a complex-conjugate pair of characteristic multipliers gets outside the unit circle the periodic solution loses stability and a quasi-periodic solution is generated, which turns into a chaotic one by increasing further the feedback gain.

\section{Quasi-Periodic Behavior}

It is readily identified by means of Poincaré map. A quasi-periodic solution can be expressed as a countable sum of periodic functions with incommensurate frequencies and its trajectory winds around a torus in state space. Since the ratio of the frequencies is irrational, the trajectory never closes on itself and

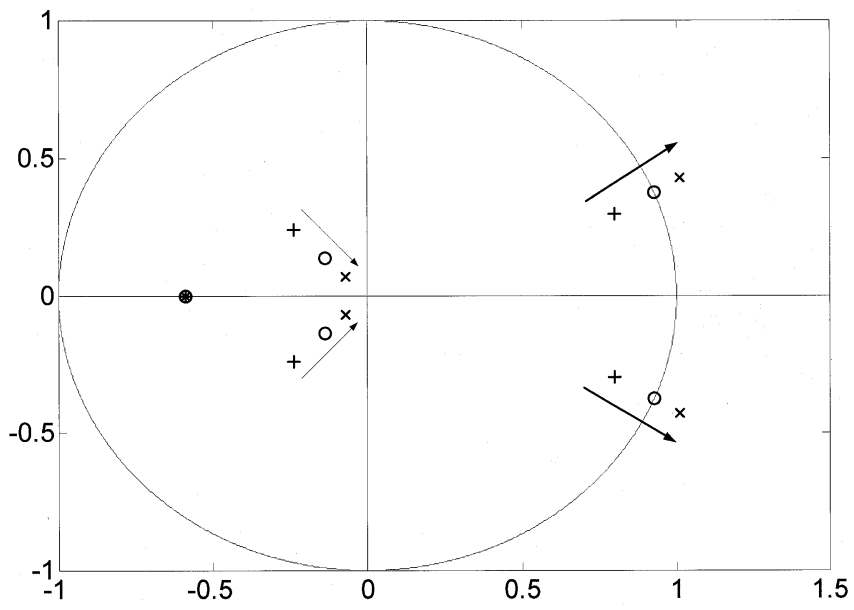

Fig. 8. Loci of characteristic multipliers as the feedback gain varies. Arrows indicate increasing feedback gain.

the torus becomes more densely filled in. The Poincaré section is in the Poincaré plane cutting through the torus, therefore the Poincaré map of the quasi-periodic trajectory is a set of separate points along a closed curve so densely populated that it looks like a curve. Fig. 9(a) shows the Poincaré map in quasi-periodic operation at $K_{V}=4$ in the reference frame of condenser voltage $v_{c k}=v_{c}\left(k T_{s}\right)$ versus output voltage $v_{o k}=v_{o}\left(k T_{s}\right)$. Similar Poincaré maps are shown in the reference frame $i_{o p k}$ vs. $v_{o k}$ in Fig. 9(b) and $i_{o p k}$ versus $v_{c k}$ in Fig. 9(c), respectively. All three Poincaré maps prove that the converter operates in quasi-periodic state.

\section{Time Functions}

The time waveforms of the condenser voltage $v_{c}$ and control voltage $v_{\text {con }}$ are plotted in Fig. 10 at $K_{V}=4$. Quasi-periodic behavior appears in systems containing two or more competing frequencies and the quasi-periodic signals may look in the time domain like an amplitude modulated waveform. The time waveform of the condenser voltage is clearly amplitude modulated [Fig. 10(a)]: the "carrier" frequency is the forcing frequency of the ramp wave; in addition, a natural frequency of the system is developed and acts as a modulating frequency. It can be seen from the succession of the peak values that the condenser voltage is not periodic. Since the two frequencies are incommensurate, the resulting waveform becomes quasi-periodic. The control voltage $v_{\text {con }}$, changing with the natural frequency of the system, hits the ramp wave once per ramp cycle. The forcing frequency of the ramp wave is present in the control voltage [Fig. 10(b)].

The quasi-periodic operation pertains approximately from $K_{V}=3.6$ up to $K_{V}=20$. Increasing further $K_{V}$ the system state turns to chaotic one, i.e., this is a route to chaos through quasi-periodic states. Fig. 11 shows the Poincaré map in chaotic behavior at $K_{V}=22$ in the reference frame of condenser voltage $v_{c k}=v_{c}\left(k T_{s}\right)$ versus output voltage $v_{o k}=v_{o}\left(k T_{s}\right)$. This chaotic map appears as set of organized points, reflecting a multilayered structure and order. The time functions of the condenser voltage $v_{c}$ and control voltage $v_{\text {con }}$ in chaotic operation are plotted in Fig. 12, at $K_{V}=22$. The control voltage fails to hit the ramp wave in its every cycle. 
TABLE II

Complex-Conjugate Pair of Characteristic Multipliers Resulting in the Hopf Bifurcation

\begin{tabular}{c|c|c|c}
\hline $\boldsymbol{K}_{\boldsymbol{V}}$ & Pair of Characteristic Multipliers & Modulus & Stability of Periodic Solution \\
\hline \hline 3 & $0.8004 \pm 0.2978 \mathrm{i}$ & 0.8540 & Asymptotically stable (period-1) \\
\hline 3.2 & $0.8428 \pm 0.3225 \mathrm{i}$ & 0.9024 & Asymptotically stable (period-1) \\
\hline 3.4 & $0.8852 \pm 0.3479 \mathrm{i}$ & 0.9511 & Asymptotically stable (period-1) \\
\hline $\mathbf{3 . 6}$ & $\mathbf{0 . 9 2 7 4} \pm \mathbf{0 . 3 7 4 0 i}$ & $\mathbf{1 . 0 0 0 0}$ & Structurally unstable (Hopf bifurcation) \\
\hline 3.8 & $0.9696 \pm 0.4008 \mathrm{i}$ & 1.0491 & Unstable (quasiperiodic) \\
\hline 4 & $1.0115 \pm 0.4282 \mathrm{i}$ & 1.0984 & Unstable (quasiperiodic) \\
\hline
\end{tabular}

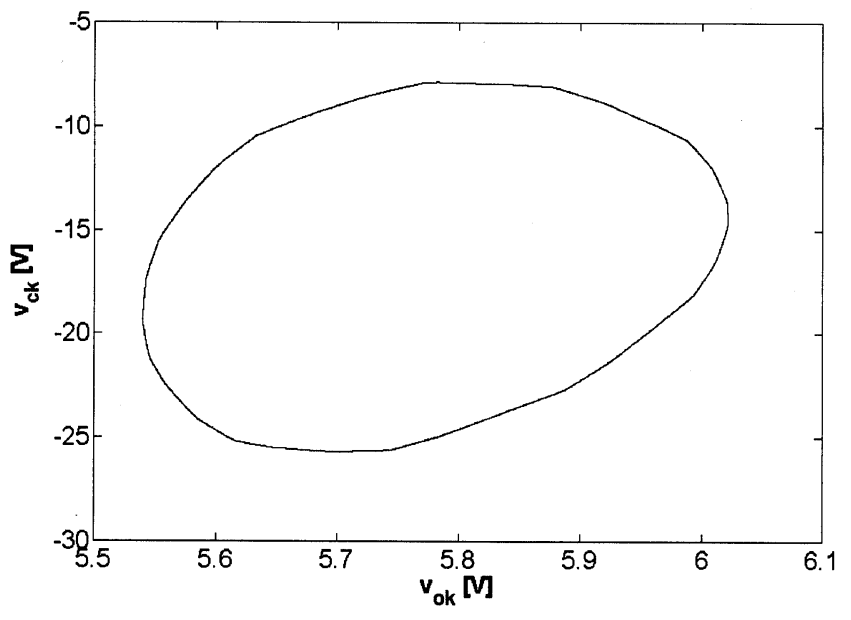

(a)

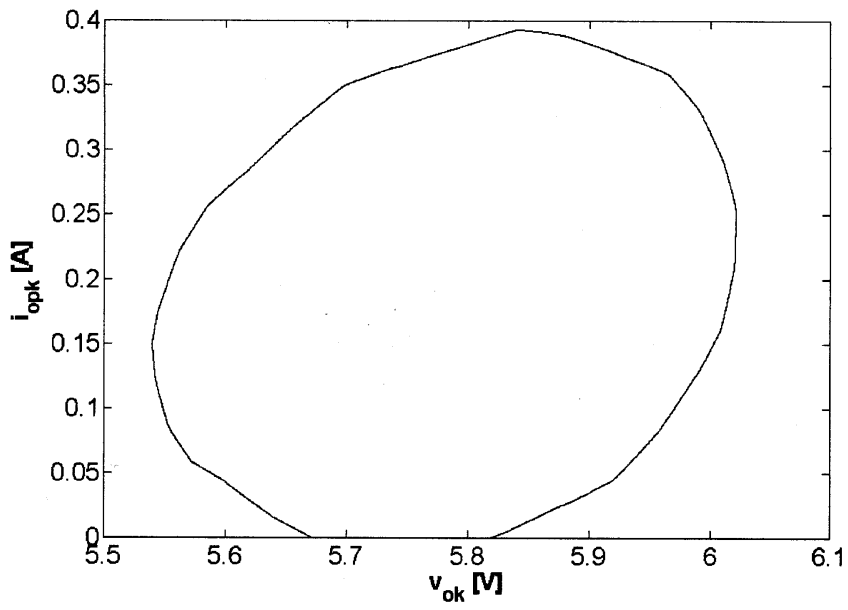

(b)

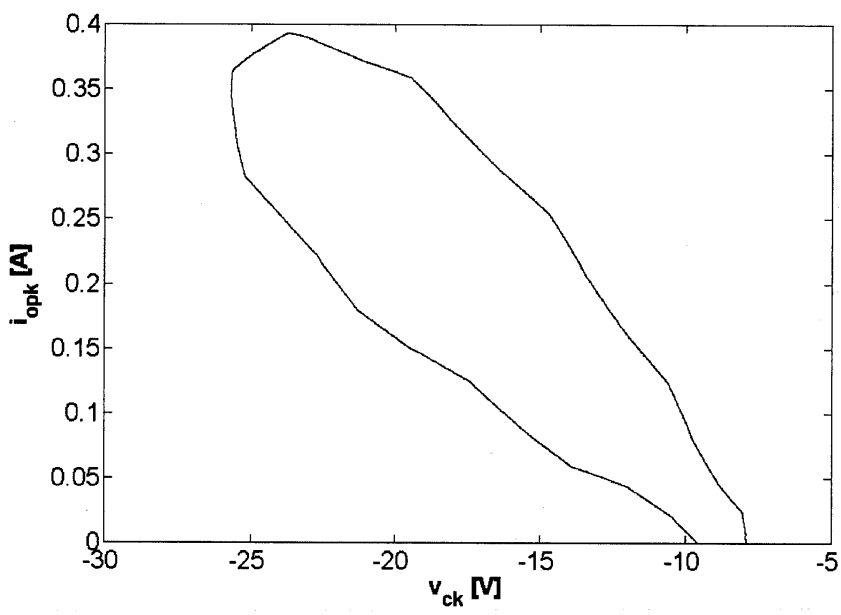

(c)

Fig. 9. Poincaré maps in quasi-periodic operation $\left(K_{V}=4\right)$.

The chaotic region suddenly changes to a periodic window about at $K_{V}=23$ (Fig. 6), followed by a cascade of period doublings. Increasing further $K_{V}$ a little above 24, chaotic state develops again. This period-doubling route to chaos was reported in detail in [5].

\section{EXPERIMENTAL RESULTS}

The values of parameters and variables used in this study are listed in Appendix B. Those ones belonging to the converter basic configuration were chosen to ensure $f_{s} / f_{r}=1$ and symmetrical CCM both in open loop $\left(K_{V}=0\right)$ and in stable feedback operation.

The oscilloscope traces of the condenser voltage $v_{c}$ and choke current $i_{o p}$ are shown in Fig. 13 at $K_{V}=3$ and in Fig. 14 at $K_{V}=8$. The choke current waveforms confirm the continuous conduction mode. The shapes of the time functions verify the results of the stability analysis performed through this paper. Quantitatively, the simulation and test results are in good agreement. The main source of deviations is the assumption of ideal components used both in the derivation of the relations and in 


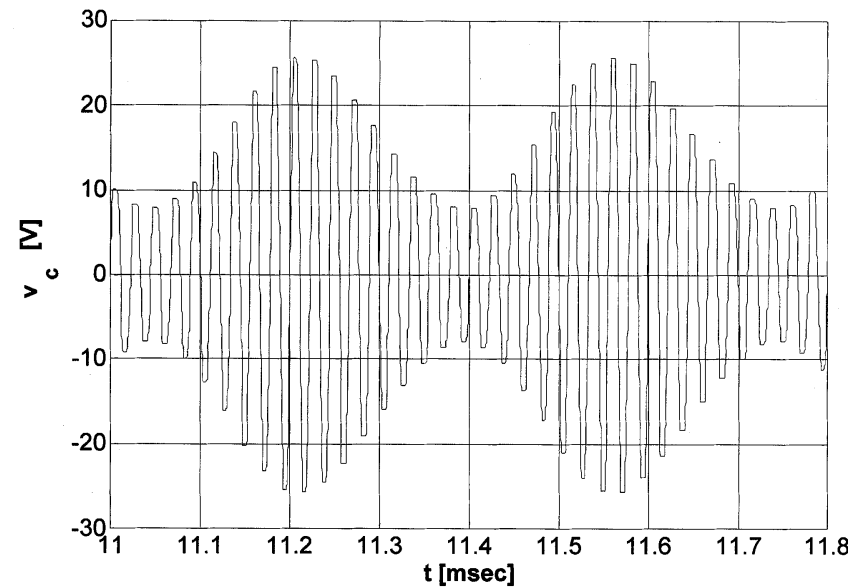

(a)

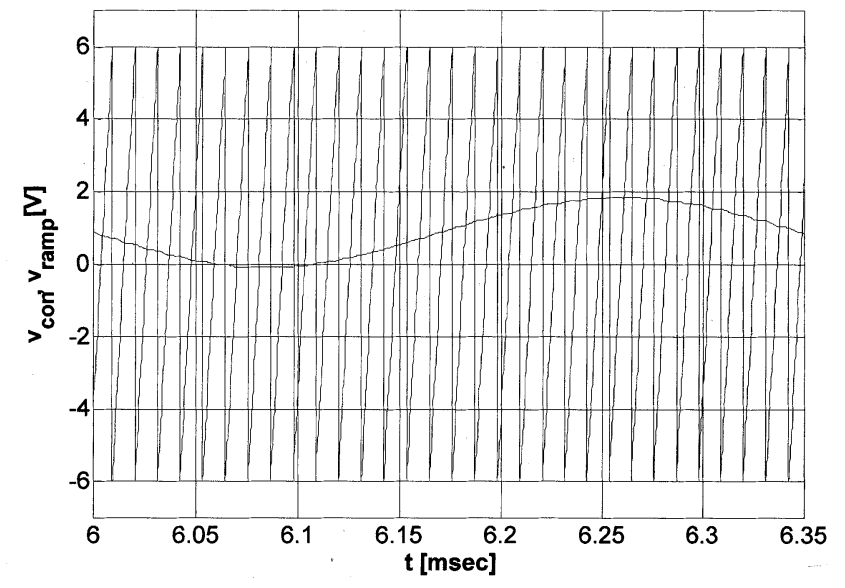

(b)

Fig. 10. (a) Condenser voltage $v_{c}$ and (b) control voltage $v_{\text {con }}$ in quasi-periodic state $\left(K_{V}=4\right)$.

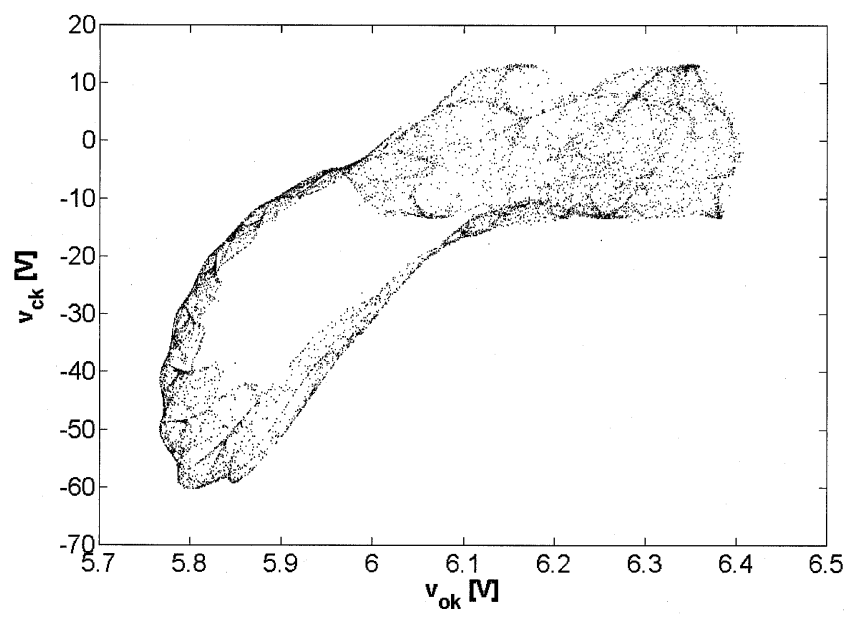

Fig. 11. Poincaré maps for chaotic operation $\left(K_{V}=22\right)$.

the simulations. Parasitic elements (the equivalent series resistance of the inductor, the forward voltages of the semiconductors) are always present in the practical circuit and they affect its behavior, but they do not affect the bifurcation structure. They were only found to shift the bifurcation points and are not essential to the phenomena reported. For example, the Hopf bi-

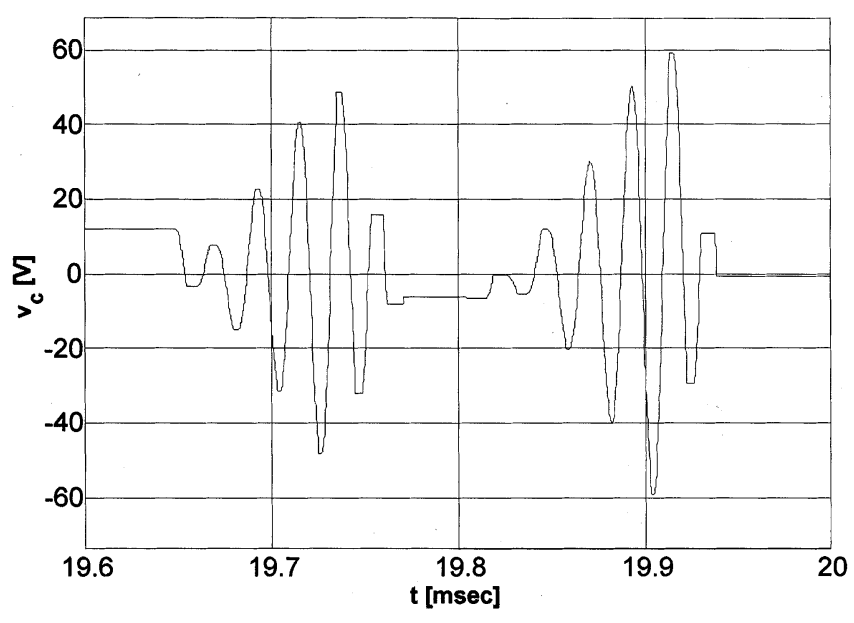

(a)

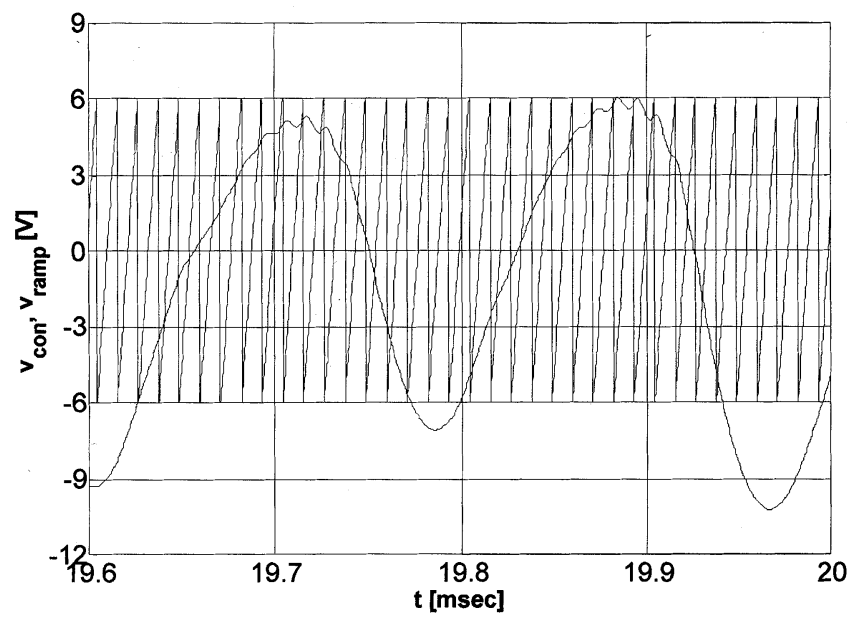

(b)

Fig. 12. (a) Condenser voltage $v_{c}$ and (b) control voltage $v_{\text {con }}$ in chaotic operation $\left(K_{V}=22\right)$.

furcation occurs in the practical circuit for an upper value of the gain, about $K_{V}=7$.

\section{CONCLUSION}

This paper has reported for the first time the stability analysis for the feedback buck member of a dual-channel resonant dc-dc converter family. The converter has five energy storage components and its output voltage is controlled by a feedback loop. The controlled switches are turned on and off by a PWM pattern using a ramp function of constant frequency. The stability of the limit cycle describing the operation of the converter is lost by increasing the loop gain and a quasi-periodic state developes. Increasing further the gain, chaotic state is reached through the quasi-periodic route. Bifurcation diagrams and Poincaré maps present the various system states.

In the stability analysis, the characteristic multipliers of the Jacobian matrix of the Poincare map function evaluated at the fixed point of the limit cycle located in the Poincaré hyperplane were determined. The stability is lost when a complex-conjugate pair of characteristic multipliers leaves the interior of the unit circle. 


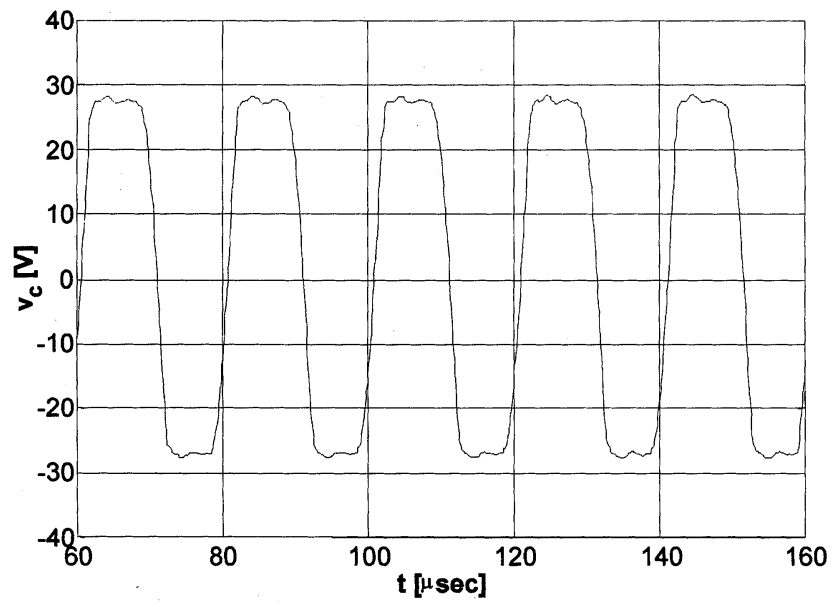

(a)

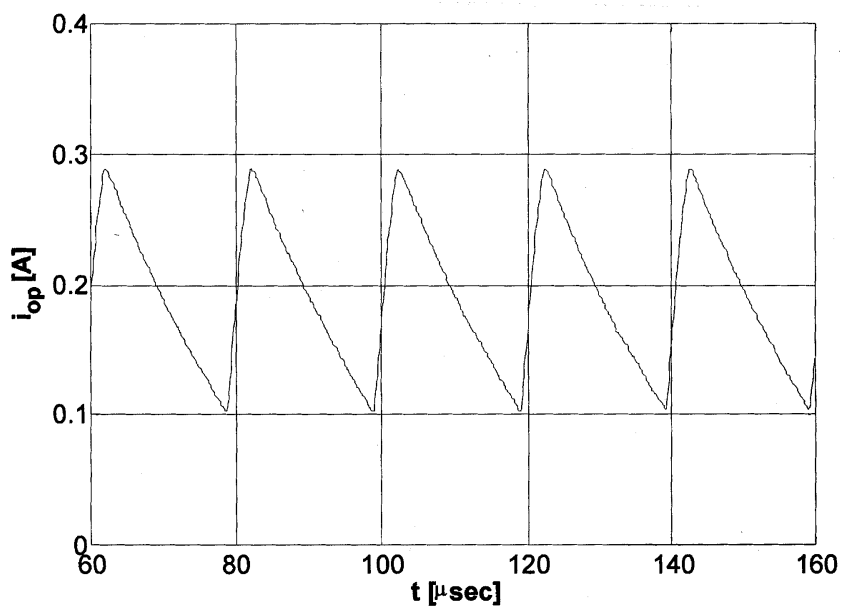

(b)

Fig. 13. Oscilloscope trace of the (a) condenser voltage and (b) choke current in period-1 operation $\left(K_{V}=3\right)$.

APPENDIX A

BASIC STEADY-STATE RELATIONS

Assuming symmetrical CCM, the current time functions in the chokes are

$$
i_{o}(\omega t)=I_{o} \cos \omega t+I_{k} \sin \omega t
$$

in the interval $0 \leq \omega t \leq \alpha$, and

$$
i_{o}(\omega t)=I_{o} \cos \alpha+I_{k} \sin \alpha-\frac{\frac{V_{o}}{2}}{Z}(\omega t-\alpha)
$$

in the interval $\alpha \leq \omega t \leq \omega T_{s}$, where $I_{o}=i_{o}(\omega t=0)$ (Fig. 2), the ripple free instantaneous output voltages are $V_{o p}=V_{o n}=$ $V_{o} / 2$ and $Z=\sqrt{L / C}$ is the characteristic impedance, furthermore,

$$
I_{k}=\frac{V_{c}-\left(\frac{V_{o}}{2}\right)+\left(\frac{V_{i}}{2}\right)}{Z}
$$

where $V_{c}=V_{c p}=-V_{c n}$ (Fig. 2) and $V_{i} / 2=V_{i p}=V_{i n}$.

The choke current is the same at $\omega t=0$ and at $\omega t=\omega T_{s}$

$$
i_{o}(\omega t=0)=i_{o}\left(\omega t=\omega T_{s}\right)=I_{o} .
$$

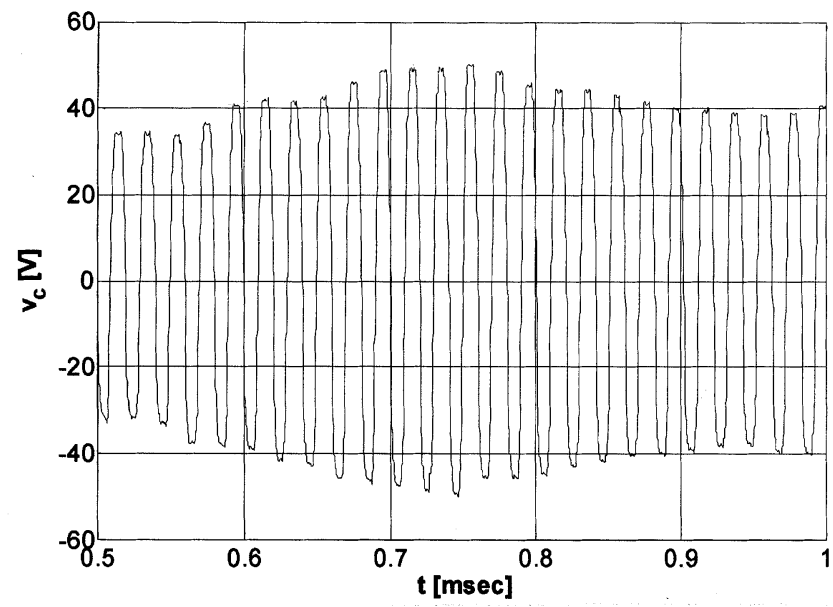

(a)

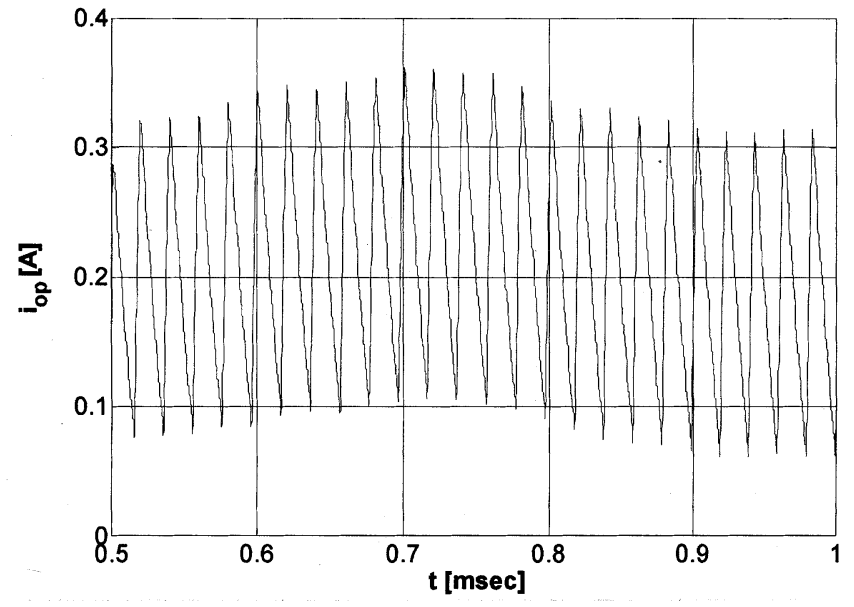

(b)

Fig. 14. Oscilloscope trace of the (a) condenser voltage and (b) choke current in quasi-periodic operation $\left(K_{V}=8\right)$.

The capacitor voltage change is the result of current $i_{c}=i_{o}$ in interval $0 \leq \omega t \leq \alpha$

$$
Z \int_{0}^{\alpha} i_{o} d(\omega t)=2 V_{c}
$$

Substituting (a1) into (a5),

$$
Z\left[I_{o} \sin \alpha+I_{k}(1-\cos \alpha)\right]=2 V_{c}
$$

and (a2) into (a4),

$$
I_{o}=\frac{I_{k} \sin \alpha-\frac{V_{o}}{2} \cdot \frac{\omega T_{s}-\alpha}{Z}}{1-\cos \alpha} .
$$

Let us substitute now $I_{O}$ from (a7) into (a6)

$$
-I_{k}+\frac{V_{c}}{Z}+\frac{V_{o}}{4 Z}\left(\omega T_{s}-\alpha\right) \frac{\sin \alpha}{1-\cos \alpha}=0 .
$$

The output voltage is on the basis of (a8) and (a3)

$$
V_{o}=\frac{V_{i}}{1+\frac{\omega T_{s}-\alpha}{2} \cdot \frac{\sin \alpha}{1-\cos \alpha}} .
$$




\section{APPENDIX B \\ PARAMETER AND VARIABLE VALUES

$$
L=125 \mu \mathrm{H} ; C=100 \mathrm{nF} ; C_{p}=C_{n}=100 \mu \mathrm{F} ; R=7 \Omega \text {; }
$$$$
V_{i p}=V_{i n}=8 \mathrm{~V} ; V_{\text {ref }}=6 \mathrm{~V} ; V_{L}=-6 \mathrm{~V} ; \text { and } V_{U}=6 \mathrm{~V} \text {. }
$$

\section{ACKNOWLEDGMENT}

The authors are thankful for the support stemming from the cooperation between the Hungarian Academy of Sciences and the Italian Scientific Research Council (CNR in Subject Number 18), and, also, thanks are extended to the Japanese Society for the Promotion of Science (JSPS).

\section{REFERENCES}

[1] I. Nagy, "Resonant DC-DC configuration," in Proc. EPE'89, Aachen, Germany, 1989, pp. 1495-1500.

[2] J. Hamar and I. Nagy, "New topologies of a resonant DC-DC converter family," in Proc. ELECTROMOTION 2001, vol. 1, Bologna, Italy, June 19-20, 2001, pp. 109-114.

[3] I. Nagy and J. Hamar, "Study of a DC-DC dual channel resonant converter," in Proc. COBEP'99, vol. 1, Parana, Brasil, Sept. 19-23, 1999, pp. 320-325.

[4] I. Nagy, P. Korondi, Z. Vranyecz, and E. Masada, "Ratio control of two DC power flows in a new resonant converter family," in Proc. IPEMC'97, vol. 2, Hangzhou, China, Nov. 3-6, 1997, pp. 609-614.

[5] I. Nagy and O. Dranga, "Bifurcation in a dual channel resonant DC-DC converter," in Proc. ISIE 2000, Dec. 2000, pp. 495-500

[6] I. Nagy, "Nonlinear dynamics in power electronics," Automatika, vol. 42, no. 3-4, pp. 117-132, July-Dec. 2001

[7] H. H. C. Iu and C. K. Tse, "Bifurcation behavior of parallel-connected buck converters," IEEE Trans. Circuits Syst. I, vol. 48, pp. 233-240, Feb. 2001.

[8] D. Carton, B. Robert, and C. Goeldel, "Self-similarity and chaos in a current mode PWM-bridge," in Proc. 6th Int. Conf. Electrimacs'99, vol. I, Lisboa, Portugal, Sept. 14-16, 1999, pp. 109-114.

[9] A. E. Aroudi, L. Benadero, E. Toribio, and S. Machiche, "Quasiperiodicity and chaos in the DC-DC buck-boost converter," Int. J. Bifurcation Chaos, vol. 10, no. 2, pp. 359-371, 2000.

[10] A. E. Aroudi, G. Olivar, L. Benadero, and E. Toribio, "Modeling PWM boost and buck-boost converters by 2-dimensional approximated nonlinear mapping," in Proc. 7th Int. Workshop Nonlinear Dynamics of Electronic Systems, Ronne, Denmark, July 15-17, 1999, pp. 95-98.

[11] T. Hashimoto, T. Ninomiya, H. Tanaka, and R. P. Tymerski, "ZVS-PWM-controlled parallel-resonant converter applied to a constant-current power supply," in Proc. IEEE PESC'99, vol. 1, 1999, pp. 275-280.

[12] K. Murata, K. Harada, and K. Ozawa, "Simplified resonant DC-DC converter with high frequency drive," in Proc. IPEC-Tokyo 2000, vol. 1, Tokyo, Japan, 2000, pp. 80-84.

[13] J. H. B. Deane, P. Ashwin, D. C. Hamill, and D. J. Jefferies, "Calculation of the periodic spectral components in a chaotic DC-DC converter," IEEE Trans. Circuits Syst. I, vol. 46, pp. 1313-1319, Nov. 1999.

[14] J. L. R. Marrero, J. M. Font, and G. C. Verghese, "Analysis of the chaotic regime for DC-DC converters under current mode control," in Proc. IEEE PESC'96, 1996, pp. 1477-1483.

[15] T. Ninomiya, A. Takeuchi, and T. Kohama, "Beat phenomenon, phaselock, and chaotic oscillation in resonant converters," in Proc. ChineseJapanese Power Electronics Conf., Sept. 1992, pp. 1-8.

[16] B. Robert, M. C. Marion-Pera, and C. Goeldel, "Dynamic behavior of a step motor analyzed by chaos theory," in Proc. 6th Int. Conf. Electrimacs 99, vol. I, Lisbon, Portugal, Sept. 14-16, 1999, pp. 115-120.

[17] J. H. Chen, K. T. Chau, and C. C. Chan, "Analysis of chaos in currentmode-controlled DC drive systems," IEEE Trans. Ind. Electron., vol. 47, pp. 67-76, Feb. 2000.
[18] J. H. Chen, K. T. Chau, S. M. Siu, and C. C. Chan, "Experimental stabilization of chaos in a voltage-mode DC drive system," IEEE Trans. Circuits Syst. I, vol. 47, pp. 1093-1095, July 2000.

[19] A. Magauer and S. Banerjee, "Bifurcations and chaos in the tolerance band PWM technique," IEEE Trans. Circuits Syst. I, vol. 47, pp. 254-259, Feb. 2000.

[20] I. Vajk, J. Hetthéssy, and H. Charaf, "Neural network approximators in modeling dynamic nonlinear systems," Electrotech. Inf. Tech., pp. $43-48,2000$.

[21] I. Vajk, "Sensitivity of nonlinear dynamic modeling," in Proc. 14th IFAC World Congr., vol. H, Beijing, P.R. China, 1999, pp. 19-24.

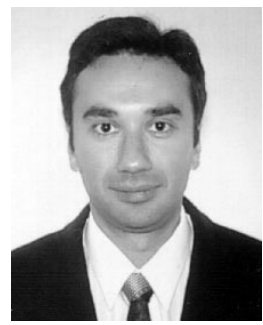

Octavian Dranga was born in Oradea, Romania, in 1971. He received the B.Sc., M.Sc., and Ph.D degrees from the Politechnica University of Timisoara, Timisoara, Romania, in 1995, 1996 and 2001, respectively. He is currently working toward the Ph.D. degree in the Department of Automation, Technical University of Budapest, Budapest, Hungary.

$\mathrm{He}$ has been associated with the Department of Automation, Politehnica University of Timisoara, for two years. His research interest is chaotic behavior in motion control. He has coauthored 15 technical papers. He is currently a Visiting Researcher at The Hong Kong Polytechnic University, Hong Kong.

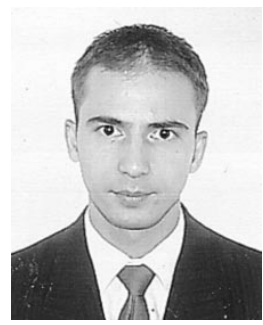

Balázs Buti He was born in Berettyóújfalu, Hungary in 1978. He received the M.Sc. degree in 2001 from Budapest University of Technology and Economics, Budapest, Hungary, where he is currently working toward the Ph.D. degree in the Department of Automation and Applied Informatics.

Since 2001, he has been associated with the Computer and Automation Research Institute of the Hungarian Academy of Sciences. His research interests are power electronics, nonlinear systems, and control systems. He has coauthored three technical papers.

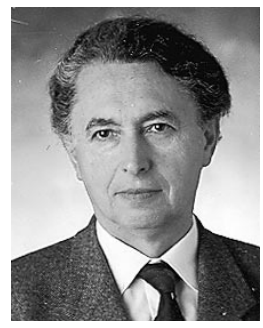

István Nagy (M'92-SM'98-F'00) graduated from and received the Ph.D. degree from the Technical University of Budapest, Budapest, Hungary, in 1953 and 1960, respectively.

$\mathrm{He}$ has been associated with the Institute of Automation and Computation of the Hungarian Academy of Sciences since 1960 and headed the Department of Power Electronics there for 15 years. He became a Full Professor at the Technical University of Budapest in 1975 and has been the Head of the Department of Electrical Engineering for 20 years. He has been a Visiting Professor at universities in Canada, Germany, India, New Zealand, Sweden, the U.S., and at The University of Tokyo, Japan. His research interests are power electronics and control of ac drives. He has authored or coauthored 12 textbooks and about 170 technical papers and is the holder of 13 patents.

Dr. Nagy is a member of the Hungarian Academy of Sciences, Hungarian Institute of Electrical Engineers, and Executive Council-European Power Electronics Association. He is also Chairman of the European Power Electronics Association-Power Electronics Motion Control Council. 